\title{
Calcium Phosphate Urolithiasis
}

National Cancer Institute

\section{Source}

National Cancer Institute. Calcium Phosphate Urolithiasis. NCI Thesaurus. Code C123243.

Urolithiasis in which the composition of the stones is predominantly calcium phosphate. 